\title{
Unsupervised Idealization of Ion Channel Recordings by Minimum Description Length: Application to Human PIEZO1-Channels
}

\author{
Radhakrishnan Gnanasambandam ${ }^{1}$, Morten S. Nielsen ${ }^{2}$, Christopher Nicolai ${ }^{1}$, \\ Frederick Sachs ${ }^{1}$, Johannes P. Hofgaard ${ }^{2}$ and Jakob K. Dreyer ${ }^{3 *}$ \\ ${ }^{1}$ Department of Physiology and Biophysics, State University of New York, Buffalo, NY, USA, ${ }^{2}$ Department of Biomedical \\ Sciences and The Danish National Research Foundation Centre for Cardiac Arrhythmia, Faculty of Health and Medical \\ Sciences, University of Copenhagen, Copenhagen, Denmark, ${ }^{3}$ Center for Neuroscience, Faculty of Health and Medical \\ Sciences, University of Copenhagen, Copenhagen, Denmark
}

Researchers can investigate the mechanistic and molecular basis of many physiological phenomena in cells by analyzing the fundamental properties of single ion channels. These analyses entail recording single channel currents and measuring current amplitudes and transition rates between conductance states. Since most electrophysiological recordings contain noise, the data analysis can proceed by idealizing the recordings to isolate the true currents from the noise. This de-noising can be accomplished with threshold crossing algorithms and Hidden Markov Models, but such procedures generally depend on inputs and supervision by the user, thus requiring some prior knowledge of underlying processes. Channels with unknown gating and/or functional sub-states and the presence in the recording of currents from uncorrelated background channels present substantial challenges to such analyses. Here we describe and characterize an idealization algorithm based on Rissanen's Minimum Description Length (MDL) Principle. This method uses minimal assumptions and idealizes ion channel recordings without requiring a detailed user input or a priori assumptions about channel conductance and kinetics. Furthermore, we demonstrate that correlation analysis of conductance steps can resolve properties of single ion channels in recordings contaminated by signals from multiple channels. We first validated our methods on simulated data defined with a range of different signalto-noise levels, and then showed that our algorithm can recover channel currents and their substates from recordings with multiple channels, even under conditions of high noise. We then tested the MDL algorithm on real experimental data from human PIEZO1 channels and found that our method revealed the presence of substates with alternate conductances.

Received: 01 July 201 Accepted: 05 April 2017 Published: 27 April 2017

Citation: Gnanasambandam R, Nielsen MS, Nicolai C, Sachs F, Hofgaard JP and

Dreyer JK (2017) Unsupervised Idealization of Ion Channel Recordings by Minimum Description Length: Application to Human PIEZO1-Channels.

Front. Neuroinform. 11:31. doi: 10.3389/fninf.2017.00031
Keywords: ion channel gating, idealization, Markov models, minimum description length, mechanoreceptors

\section{INTRODUCTION}

The analysis of discrete events in single ion channel data has been a powerful tool in electrophysiology research since the pioneering work of Erwin Neher and Bert Sakmann, recognized with the 1991 Nobel Prize in Medicine. However, these analyses usually entail data modeling methods that rely on user-defined inputs, filters, event detection thresholds or subjective 
criteria for event detection. These conditions present difficulties, particularly when analyzing time series composed of currents from multiple ion channels, or in cases when a channel can make a transition between one or more sub-conductance states.

Given these considerations, approaches to the analysis of ion channel currents often involve one of two paradigms. In one approach, Hidden Markov Models (HMMs) are used to analyze recordings containing signals arising from one or more ion channels (Venkataramanan and Sigworth, 2002; Qin, 2007). Here, we assume that the ion channels generate the observed currents by jumping between metastable conductance states. Consequently, HMM analysis is most suitable when different conductance states of channels can be estimated a priori; so long as these estimates are valid, the HMM algorithm provides maximal information about the conductance transitions and their kinetics.

HMM is not always applicable, precisely because it requires $a$ priori knowledge of likely kinetic models, which can be especially difficult to predict if currents from several channels are present in the recording. While the HMM-based algorithms can provide useful quantitative analyses, real laboratory data often fail to satisfy the inherent assumptions of the model. Chief among these assumptions or conditions is that the system should be in a steady-state for the duration of the recording. However, the channel kinetics and ion flux can vary with time, for example the membrane resting potential may change during the recording (Suchyna et al., 2009; Gottlieb et al., 2012). An alternative to the HMM approach is to idealize the measured input sequence using an event detection algorithm that is independent of the kinetic model (Colquhoun and Sigworth, 1995; Vandongen, 1996; Carter et al., 2008; Parsons and Huizinga, 2013). Here events are detected when the signal, or its first derivative, crosses a certain threshold. The measured current is thus modeled as a series of events without imposition of a particular kinetic scheme, and can in principle account for unknown sub-states in the recording (Vandongen, 1996). However, such methods usually require low-pass filtering to reduce noise levels. In principle, the optimal filtering can be deduced from the signal to noise ratio and methods that analyze the filtered time course of conductance transitions enable highly accurate determination of transition points (Colquhoun and Sakmann, 1985). However when the number of subconductance levels and their kinetics is unknown, low pass filtering may lead to missed events.

Hotz et al. (2013) have used a jump-segmentation filter to analyze gramicidin A channels (J-SMURF) (Hotz et al., 2013). This method uses a statistical multiresolution analysis and detects abrupt changes in a recording, and does not assume a specific channel structure. The sensitivity of the method is controlled by the user by setting a significance level, say 5\%, which corresponds to the risk of false positive segmentation. However, only few methods enable idealization of ion-channel recordings completely without inputs from the user.

We now test Rissanen's Minimum Description Length Principle (MDL) to provide an idealization algorithm based on "structural breakpoint detection" (Rissanen, 1978; Lee, 2001; Davis et al., 2006; Killick et al., 2012). Our aim is to provide a fast and unbiased idealization of single channel time-series without requiring any user-dependent inputs. We assume that the current can be modeled stepwise as a sequence of segments of constant amplitude, separated by abrupt (instantaneous) transitions to some new current amplitude (Vandongen, 1996; Hotz et al., 2013). The algorithm identifies the location of the transitions between segments, and calculates the mean current prevailing in each segment. Furthermore, the MDL algorithm performs the calculation of transition locations and amplitudes without a priori assumptions of step-size or transition kinetics, and the current amplitude in each segment is independent of other amplitudes occurring in the recording. To provide a post-hoc analysis of the output data, we developed a correlation analysis in which the conditional probability of observing pairwise adjacent steps is used to infer the number of discrete states. Finally, we tested the MDL algorithm on real experimental data from human PIEZO1 channels and found that our method revealed the presence of substates with alternate conductances.

\section{METHODS}

\section{Cell Culture and Electrophysiology}

We analyzed current records from the mechanically gated channel PIEZO1 in transformed HEK cells maintained in an incubator at $37^{\circ} \mathrm{C}$ and $5 \% \mathrm{CO}_{2}$. These cells had been transiently transfected with 200-500 ng of hPIEZO1 cDNA using Mirus $^{\text {TM }}$ (TransIT-293 reagent) 1-2 days prior to performing cell-attached patch-clamp recordings at room temperature. The resting membrane potential of cells was maintained close to 0 $\mathrm{mV}$ by using a high potassium bath solution containing $150 \mathrm{mM}$ $\mathrm{KCl}, 10 \mathrm{mM}$ HEPES, and $1 \mathrm{mM} \mathrm{MgCl}$ and $\mathrm{CaCl}_{2}$, adjusted to $\mathrm{pH}$ 7.4. The pipette solution contained $150 \mathrm{mM} \mathrm{KCl,} 80 \mathrm{mM}$ TEA, and $10 \mathrm{mM}$ HEPES at $\mathrm{pH}$ 7.4. Recording was performed in the cell-attached configuration in which the final membrane potential $\left(V_{m}\right)$ of the patch would be $V_{c}-V_{p}$ (where $V_{c}$ and $V_{p}$ are the resting cell membrane potential and the command potential, respectively). $V_{m}$ would be roughly equal in magnitude and opposite in polarity to the command potential $\left(V_{p}\right)$ when resting membrane potential is roughly $0 \mathrm{mV}$, as set by the high potassium bath. During the recordings, the patch membrane potential was randomly stepped to voltages within the range of $-100 \mathrm{mV}$ to $+100 \mathrm{mV}$ and pressure steps were applied to the pipette using a high-speed pressure clamp (ALA Scientific) (Figures 6A,B). Data sampling was at $10 \mathrm{kHz}$, with Bessel filtration at $2 \mathrm{kHz}$.

\section{Mathematical Analysis}

The basic assumption in our method is that the opening and closing of an ion channel gives rise to instantaneous changes in membrane current, which are superimposed on a noisy background of the recording. Accordingly, we modeled the time series of currents as a sequence of steps corresponding to opening and closing of channels and additive Gaussian noise (ignoring for the present the possibility of state-dependent noise). We define time points at which the conductance jumps occur as break points, and the difference in mean amplitude on each side of the break point as step height (or step amplitude).

Consider a data set denoted as $\boldsymbol{x}=\left(x_{1}, x_{2}, \ldots, x_{N}\right)$ consisting of $N$ data points. Here, we define the code length $L$ as the length of 
the message containing the relevant information in the data. The code length, $L$, need not be constant, but varies depending on how the information in $\boldsymbol{x}$ is represented. More effective coding schemes generally have low description length. In particular, if $\boldsymbol{x}$ is well described by a particular model, it may be more efficient to first encode the model parameters, and then encode any deviations from the model (Rissanen, 1978; Lee, 2001; Davis et al., 2006). MDL serves to rank the fitness of models of a data set by identifying the particular model producing the shortest total description length for the observations; in other words, the task is to select the model providing an optimal tradeoff between model complexity and fitting of the data.

In order to apply MDL to an ion channel recording, we first need to calculate the lower bound of the description length of the observed time series, $\boldsymbol{x}$. Considering a time series segment $\boldsymbol{x}=$ $\left(x_{1}, x_{2}, \ldots, x_{N}\right)$ with mean value $\mu$, the residual sum of squares (RSS) is defined as

$$
R S S_{0}=\sum_{i=1}^{N}\left(x_{i}-\mu\right)^{2}
$$

We now consider the minimum description length for encoding the data sequence (Rissanen, 1978; Lee, 2001). A set of $N$ data points with a Gaussian distribution can be most efficiently encoded as the mean value and a list of deviations from the mean. Thus, the minimum description length of a segment described by a single mean value is given as

$$
L_{0}=\frac{1}{2} \log (N)+\frac{N}{2} \log \left(\frac{R S S_{0}}{N}\right)
$$

In Equation (2) the first term is the description length of the mean value; the minimum number of bits required to encode the mean value depends on the precision with which it is determined, and that precision is determined by $N$, the number of data points. The second term of the equation is the description length of the $N$ deviations from the mean. By convention of information theory, base 2 logarithms $\left(\log _{2}\right)$ are usually used to calculate code length in units of bits. However, for the application at hand, we are interested in comparing relative changes in code length, and therefore prefer to use the natural logarithm, the particular base being irrelevant.

We next consider the same sequence of data, but divided into daughter segments at $k$ locations defined by $2 \leq n_{i} \leq N-1$, and where $n_{i}<n_{i+1}$. We then define the residual sum of squares of the divided segment $\left(\mathrm{RSS}_{k}\right)$ as

$$
\operatorname{RSS}_{k}\left(\left\{n_{1}, \ldots, n_{k}\right\}\right)=\sum_{i=1}^{k+1} \sum_{j=1}^{N_{i}}\left(x_{n_{i-1}+j}-\mu_{i}\right)^{2}
$$

where $n_{i}$ is the $i$ th division point and $\mu_{i}$ is the mean value of the $N_{i}$ data points between $n_{i-1}$ and $n_{i}$. As above, we then calculate the minimum description length of the divided segment as (Lee,
2001),

$$
\begin{aligned}
L_{k}\left(\left\{n_{i}, \ldots, n_{k}\right\}\right)= & \frac{k}{2} \log (N)+\frac{1}{2} \sum_{i=1}^{k+1} \log \left(N_{i}\right) \\
& +\frac{N}{2} \log \left(\frac{R S S_{k}}{N}\right)
\end{aligned}
$$

Here the first term describes the code length for the location in the data string of the defined break points, i.e., the points at which the segment is divided into $k+1$ daughter segments. The second term describes the code length of the mean value of each segment, and the third term is the code length for the residuals. According to the Minimal Description Length Principle, a divided segment can be considered a better model of the data only if $L_{k}<L_{0}$. In our implementation, we applied Equation (4) with $k=1$ or $k=2$.

\section{Step Detection Algorithm}

Having come this far, the task is now to identify a set of breakpoints minimizing the description length of a given dataset. This is inherently a complex multidimensional optimization problem. For typical data the number of possible segmentations is enormous and a full search for all possible breakpoints not feasible. We therefore search for breakpoints iteratively using a modification of the Binary Segmentation process (Scott and Knott, 1974; Kalafut and Visscher, 2008). In brief, we try to locate 1 or 2 breakpoints in the full segment and, if successful, we repeat the search on each sub-segment. We first try to locate a single break point in the time series. If this putative breakpoint fails to reach the MDL criterion, we try to locate two break points. Thus, our search is essentially a tertiary segmentation process with an initial binary search step. Using numerical tests, we show below that for the binary search, the probability of detecting channel-like events depends on the recording length, but that this undesirable property is circumvented with the tertiary search.

The algorithm proceeds in the following steps:

(1) Calculate $L_{0}$ for the segment.

(2) Search for the optimal location for a single break point $(k=$ $1)$. In other words, find $n^{*}$ as the $n$ where $R S S_{1}$ is minimized. Thus, we define $n^{*}$ as

$$
n^{*}=\arg \min \left(R S S_{1},\left\{n_{i} \mid l_{\min }<i \wedge i<N-l_{\min }\right\}\right)
$$

where $l_{\text {min }}$ is a cutoff threshold for the smallest segment allowed. Unless otherwise stated we use $l_{\min }=3$.

(3) Apply MDL to test whether division at the optimal breakpoint, $n^{*}$, is indeed a better model than for the entire undivided segment:

a. Calculate $L_{1}\left(n^{*}\right)$ using Equation (4)

b. If $L_{1}\left(n^{*}\right)<L_{0}$ the segment is divided at $n^{*}$ and the procedure is repeated from step 1 for each of the two daughter segments.

Thus, if the single breakpoint is accepted in step $3 \mathrm{~b}$, the algorithm is repeated from step 1. Otherwise, we proceed by evoking the tertiary search noted above, thus trying to locate two breakpoints in the segment: 
(4) Find the pair $\left\{m_{1}^{*}, m_{2}^{*}\right\}$ for which $R S S_{2}$ is minimized.

(5) Apply MDL to test if a model with divisions at the two breakpoints, $\left\{m_{1}^{*}, m_{2}^{*}\right\}$, provides a better model than the undivided segment:

a) Calculate $L_{2}\left(\left\{m_{1}^{*}, m_{2}^{*}\right\}\right)$ using Equation (4)

b) Accept $\left\{m_{1}^{*}, m_{2}^{*}\right\}$ as break points if $L_{2}\left(\left\{m_{1}^{*}, m_{2}^{*}\right\}\right)<L_{0}$

(6) If the breakpoints are accepted, we repeat the procedure from step 1 onward for each daughter segment.

The algorithm proceeds until no sub-segment is further divisible (into two or three parts). Ultimately, the algorithm produces a list of breakpoints and the mean value of the segments between break points follows easily.

The number of calculations using the tertiary search in step 4 scales with $N^{2}$. However, in data containing many breaks, the binary search, i.e., steps $1-3 . b$ of the search algorithm, is often by itself sufficient to decompose the sequence into shorter segments. This reduces the total computing time because the computationally demanding tertiary search is only evoked on shorter sub-segments later in the search (see Figure 3).

We tested a number of alternate criteria for the detection of break points. Similar results were obtained when putative break points were tested using the Bayes Inference Criterion (BIC) (Schwarz, 1978), although a minimum segment length $\left(l_{\min }=10\right)$ was required to avoid false segmentation of small segments. The Akaike Information Criterion (AIC) (Akaike, 1973) allowed many false steps, and was thus considered inappropriate in the present application (data for BIC and AIC are not shown). Similar observations have been reported by Kalafut and Visscher (2008), who used BIC in their event detection algorithm. We note that the alternate criteria BIC and AIC both require a priori knowledge of the variance of the noise. While this is estimable from a segment of the time series in which no steps/transitions were apparent by visual inspection, such a manual approach introduces bias favoring higher baseline noise models. However, the MDL method does not require this potentially subjective operation, and has superior performance in terms of low number of false positives.

The step detection algorithm with MDL was implemented in MATLAB and is available on MATLAB central file exchange (Dreyer, 2016). It is also available as a plugin for ionchannel analysis software QuB (https://www.qub.buffalo.edu/ download/).

\section{Computational Complexity}

The RSS of $N$ points was computed with $O(N)$ time complexity, using a well-known incremental one-pass method (Chan et al., 1983).

$$
\begin{aligned}
\mu_{i} & =\frac{1}{i}\left[(i-1) \mu_{i-1}+x_{i}\right] \\
R_{R S} & =R_{i} S_{i-1}+\left(x_{i}-\mu_{i-1}\right)^{2}-i\left(\mu_{i}-\mu_{i-1}\right)^{2}
\end{aligned}
$$

We derived the decrimental form, which removes a point from the distribution by solving for $\mu_{i-1}$ and $R S S_{i-1}$.

$$
\begin{aligned}
\mu_{i-1} & =\frac{1}{i-1}\left(i \mu_{i}-x_{i}\right) \\
R S S_{i-1} & =R S S_{i}-\left(x_{i}-\mu_{i-1}\right)^{2}+i\left(\mu_{i}-\mu_{i-1}\right)^{2}
\end{aligned}
$$

With these methods, the optimal break point, $n^{*}$, was found with $O(N)$ complexity. We initialized the left-hand distribution $D_{L}$ with the first $l_{\min }$ points, and the right-hand distribution $D_{R}$ with all $N$ points. The $R S S_{2}$ of the first break point candidate is then the sum of the left- and the right-hand incremental RSS values. We computed the $R S S_{2}$ of each subsequent candidate by adding a point to $D_{L}$ and removing it from $D_{R}$. The tertiary search for the pair $\left\{m_{1}^{*}, m_{2}^{*}\right\}$ was implemented by evaluating, for all possible $\mathrm{m}_{1}$, the optimal binary division of points $m_{1 \ldots N}$, thus meeting the criterion for $O\left(N^{2}\right)$ complexity.

The overall complexity of the algorithm depends on the scale of the input data and the order in which intervals are segmented. Considering for example the binary segmentation of an interval sequence $\mathrm{ABCDEFGH}$ (i.e., $N_{I}=8$ intervals), in the best possible case this might be decomposed first as $\mathrm{ABCD}, \mathrm{EFGH}$, then $\mathrm{AB}$, $\mathrm{CD}, \mathrm{EF}, \mathrm{GH}$, and finally A, B, C, D, E, F, G, H. The tree's height, or in other words the number of generations, is then $g=\log _{2}\left(N_{I}\right)$. In the worst case, the sequence might be first decomposed into A, BCDEFGH, then A, B, CDEFGH, and so on, and $g=N_{I}-1$, or $O\left(N_{I}\right)$. Since each generation involves a linear pass through the data, the intervals will be fully split with $O(g N)$ complexity. If signal-to-noise ratio (SNR) is low, intervals are found by the tertiary search, which increases the complexity to $O\left(g N^{2}\right)$. Thus, in many applications, the SNR is a critical factor influencing computational burden; when SNR is high, most steps are found using a single point search and computation time grows like $O(g N)$, but when SNR is low, double point search dominates, such that computation time grows like $O\left(g N^{2}\right)$.

We tested the computation time of the MDL algorithm and compared with timing of HMM based segmentation using "viterbi_path," a MATLAB implementation of the Viterbi algorithm coded by Murphy (1998). We performed the test on a Macbook pro (2.3 GHz Intel Core i7) using the builtin MATLAB timer functions "tic" and "toc." Timing of the R-process JSMURF was done by measuring the user-CPU-time using proc.time() on the same computer as the MATLAB tests. We took care to ensure that the process used around $100 \%$ of the resources in a single CPU core.

\section{Coherence Spectra}

We determined the coherence of the idealized output of the sequence with the input sequence as a function of noise level (Figure 2). At each defined SNR, we generated five data sequences, each consisting of 131,072 data points, using a single Markov process. We average the coherence spectra in sections of 1,024 points with $50 \%$ overlap and Hamming window using the MATLAB function "mscohere."

\section{Correlation between Neighboring Events}

The outputs of our algorithm are a list of breakpoints indicating the locations where the current is found to change abruptly, and the step amplitudes at these locations. Since the breakpoints and step amplitudes are determined independently, one therefore 
needs to undertake post-processing in order to analyze properties of single channels, in particular when there are transitions between states of various condutance.

We define the step amplitude $s_{i}$ is defined as $\mu_{i+1}-\mu_{i}$. The precision of $s_{i}$ compared to the true value is given by

$$
\beta=\frac{l_{i}+l_{i+1}}{\sigma^{2}}
$$

where $l_{i+1}$ and $l_{i}$ is the length of each of the adjacent segments and $\sigma^{2}$ is the variance of the noise.

Consider a list of $M$ step amplitudes, $s_{1}, s_{2}, \ldots, s_{M}$ determined from an ion channel recording. We denote by $P(x)$ the probability of observing a step of size $x$, and calculate this probablity as

$$
P(x)=\frac{n\left(s_{j}=x \pm \frac{\Delta}{2}\right)}{M}
$$

Here, the numerator indicates the number of steps with amplitude $\mathrm{x}$ and within a bin-width $\Delta$, and the denominator is the total number of observed steps.

Our aim is to determine how often steps of amplitude $x$ were immediately followed by steps of amplitude $y$ in the recording. To this end, we therefore first define $P_{x y}$ as the joint probability of observing a step of amplitude $x$ being immediately followed by a step of amplitude $y$ :

$$
P_{x y}=\frac{n\left(s_{j}=x \pm \frac{\Delta}{2} \bigcap s_{j+1}=y \pm \frac{\Delta}{2}\right)}{M}
$$

Here the numerator indicates the number of steps with amplitude $\mathrm{x} \pm \Delta / 2$ followed by steps of amplitude $\mathrm{y} \pm \Delta / 2$. The correlation between subsequent neighboring steps was then calculated as

$$
C_{x y}=P_{x y}-P(x) P(y)
$$

The sign of $\mathrm{C}$ indicates if particular transitions are over- or underrepresented relative to an independent process, and may thus provide physiologically relevant details of the underlying step-generating mechanism. In particular, $C_{x y}>0$ if the observation "step of size $x$ is followed by step of size $y$ " happens more frequently than if the observed sequence was purely random and $C_{x y}<0$ if it is less frequent. We used the MATLAB function "hist3" to generate joint probability histograms, selecting a bin width $\Delta$ in the range $5-10 \%$ of the typical maximal amplitude. The resultant joint probability distributions, $P_{x y}$ and $C_{x y}$, were smoothed using a 2D Gaussian kernel with a half-maximum width of 1 bin-width. If either $P(x)$ $=0$ or $P(y)=0$ (that is to say, if no events occurred with amplitudes $x \pm \Delta / 2$ or $y \pm \Delta / 2$ ), the correlation is undefined. In the analysis below we consider regions where the bin counts in the joint probability histogram $\leq 1$ as undefined and these regions are indicated by white in the plots.

\section{Generation of Simulated Data}

We used simulated data for evaluating the basic properties of our step detection method (Figures 1-5). As test data for quantifying the reliability of our detection mechanism, we simulated a simple

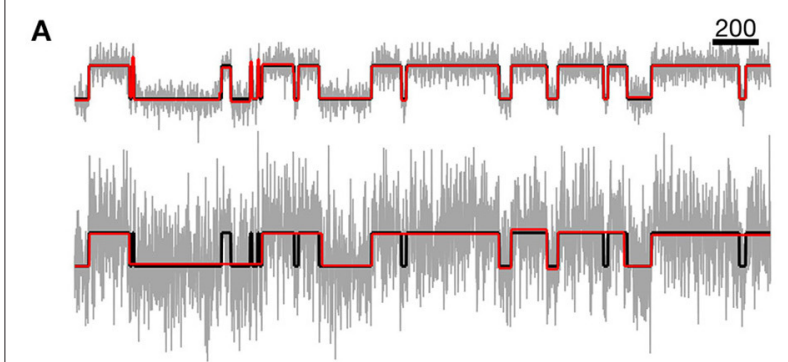

B

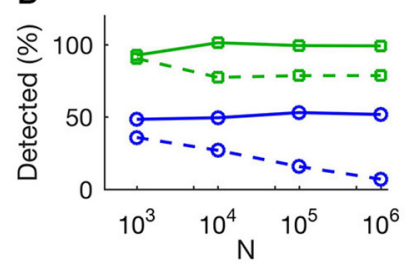

D

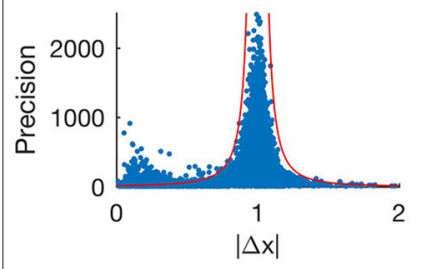

$\mathbf{F}$
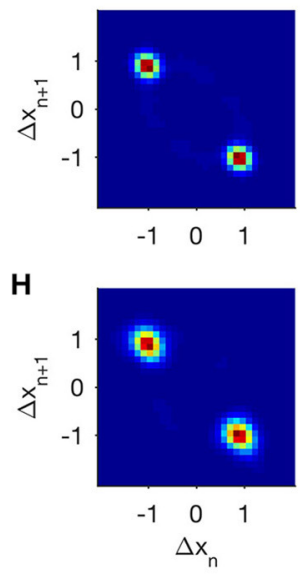

C

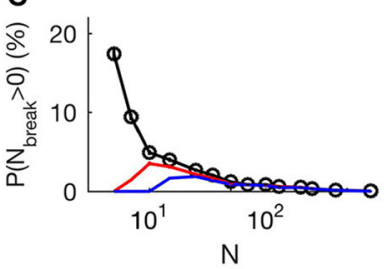

E

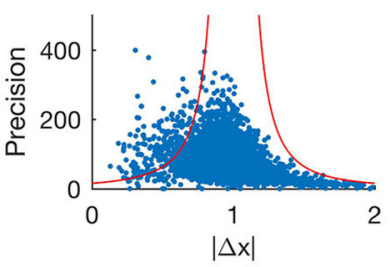

G

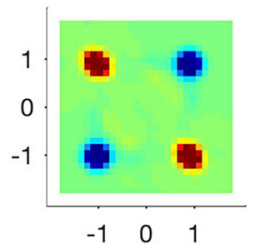

I

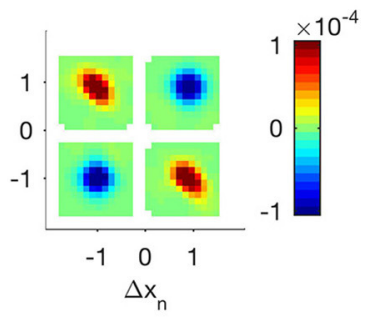

FIGURE 1 | Evaluation of the step detection method applied to synthetic data. (A) 1,000 data points of unit steps (upper, SNR = 3.3; lower, $\mathrm{SNR}=1$ ). Input data are shown in black, MDL-idealized data are shown in green, and true states are shown in red (note that the green and red lines often coincide). (B) The tertiary search method provides a constant frequency of event detection regardless of recording segment length. Markers show the number of detected steps relative to the number of known steps as a function of $N$, the number of data points in the record. Squares (green).: SNR $=3.3$ Circles (blue): SNR $=1$. Solid lines show results from the tertiary search, and dashed lines show the results from the binary search. (C) Analysis of probability of false events. Each data point shows mean number of false events from 5,000 sequences of uniform random numbers of length $N$. Black: $I_{\min }=1$, red: $I_{\min }=3$, and blue: $I_{\min }=5$. (D,E) Analysis of precision in step estimates at SNR 3.3 and 1 respectively. Dots show precision of jump estimate as function of observed absolute jump. We calculated the precision using the length of the adjacent segments according to Equation (8). Red lines indicate 4 standard deviations from the target value at different levels of precision.

(Continued 


\section{FIGURE 1 | Continued}

(F): Histogram of neighboring events at SNR 3.3. The $x$-axis shows $\Delta x$ of event $n$ and the $y$-axis shows $\Delta x$ of the following event, $n+1$. (G): Correlation of events at SNR 3.3. Detected events display same correlations of neighboring events as a simple channel. Red indicates neighboring events occurring more frequently than random. Blue indicates neighboring events occurring less frequently than random. White areas indicate undefined correlation. (H): Histogram of neighboring events at SNR 1. (I): Correlation of events at SNR 1.

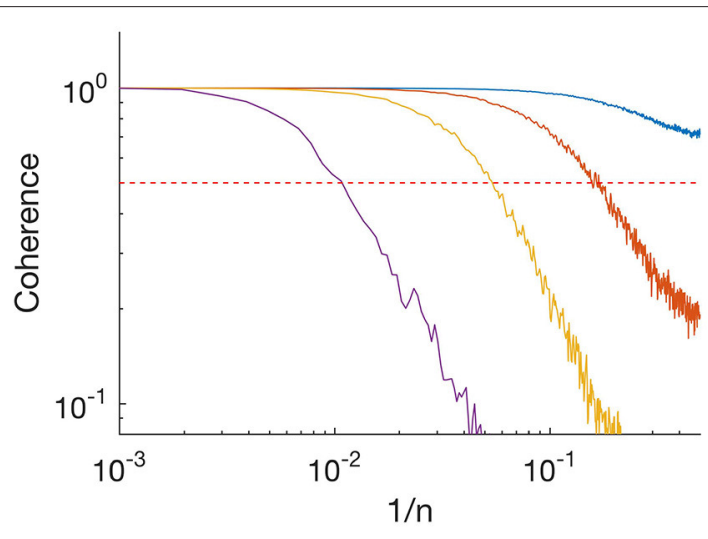

FIGURE 2 | Absolute value of coherence spectrum between input time series and idealized time series. Purple, $S N R=1$; yellow, $S N R=3.3$; orange, $\mathrm{SNR}=10$; and blue, $\mathrm{SNR}=33$. Frequency is plotted as $1 / n$ where $n$ is the length of the segment. Horizontal red dashed line indicates 0.5 .

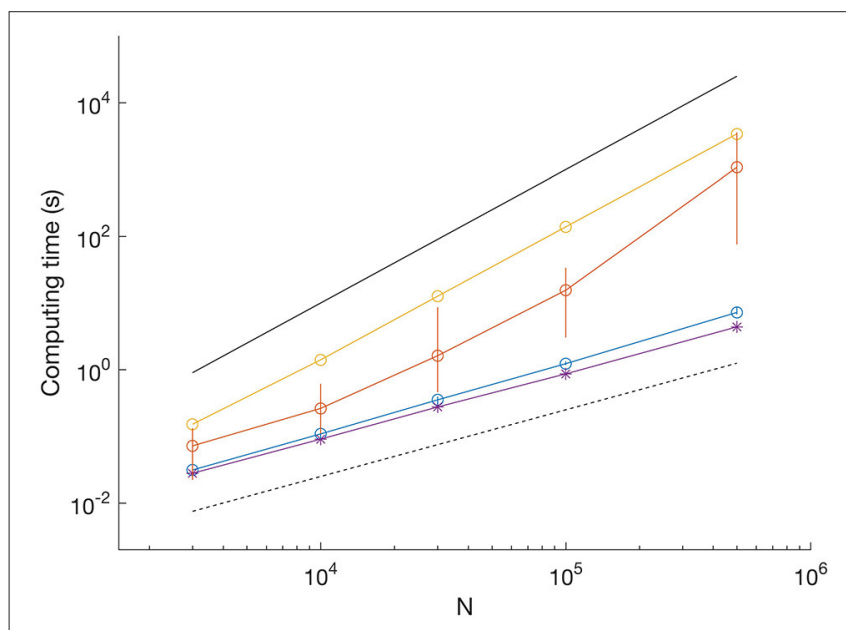

FIGURE 3 | Comparison of computation time for different datasets. $S N R=3.3$; blue dots show mean and vertical lines show range between minimum and maximum from 10 iterations. SNR $=1$; orange dots show mean and vertical lines show range from 10 iterations. SNR $=0$ (uniform random data), yellow, single iteration. Example of scaling by Viterbi algorithm $(S N R=3.3)$; purple asterisk, single iteration. Black lines indicate $O(N)$ (dashed) and $O\left(N^{2}\right)$ scaling (solid).

two-state ion channel with the transition probability between conduction states being 1/100 per time step (Figure 1). The kinetics and noise in the test data were deliberately defined so as to challenge the limits of our detection method. Test
A
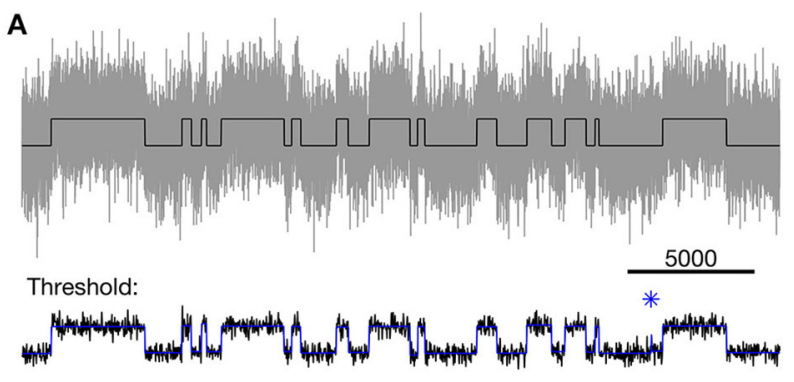

HMM:

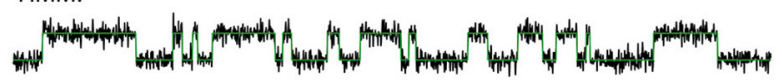

J-SMURF: MDL:

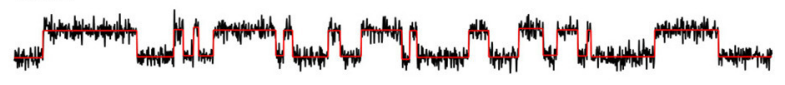

B
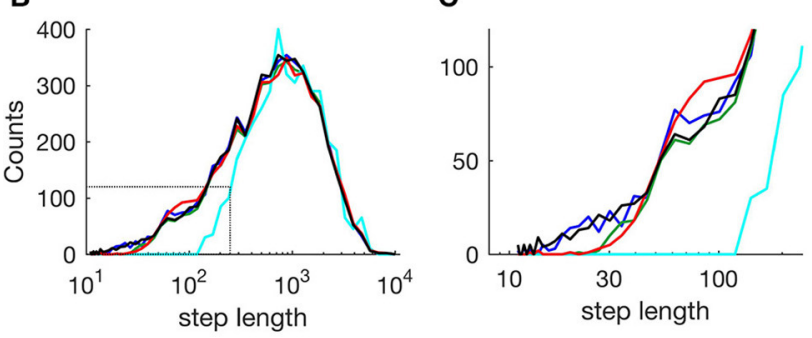

D
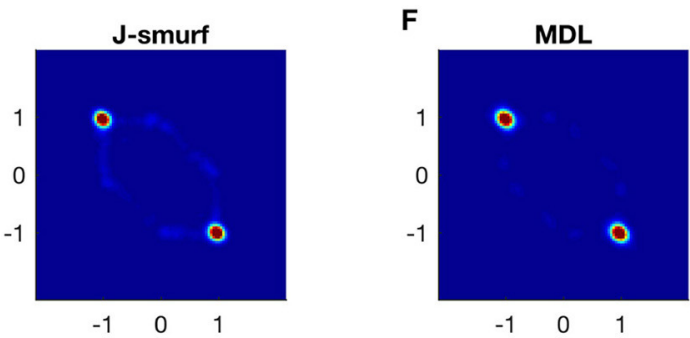

E

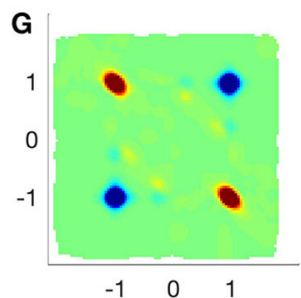

FIGURE 4 | Event detection using different algorithms on simple simulated data. (A) Top; part of the raw input data (gray) and Markov states (black); lower panels show states detected using different methods (input data are smoothed for easier comparison). Blue: states detected using threshold crossing algorithm applied on low-pass filtered data. Asterisk indicates a false detection. Green: states detected using Viterbi-algorithm as implemented in QuB. Cyan: states detected using J-SMURF. Two asterisks indicate false segmentations. Red: states detected using MDL-algorithm. (B) Distribution of step-lengths in logarithmic bins. Black, step lengths of input; blue, threshold crossing on optimally low-pass filtered data $\left(F_{N y} / 40\right)$; green, Viterbi; red, MDL; cyan, J-SMURF (rescaled). Black dashed lines indicate regions highlighted in (C). (C) Close-up view of distribution of step lengths, colors as in (B).

(Continued) 


\section{FIGURE 4 | Continued}

(D) Histogram of neighboring events detected by J-SMURF. The $x$-axis shows $\Delta x$ of event $n$ and the $y$-axis shows $\Delta x$ of the following event, $n+1$. (E) Correlation of events detected by J-SMURF. Red indicates neighboring events occurring more frequently than random. Blue indicates neighboring events occurring less frequently than random. White areas indicate undefined correlation. (F) Histogram of neighboring events detected by J-SMURF. (G) Correlation of events detected by MDL.

data for quantifying the false positive detections consisted of homogeneous sequences of random numbers with a Gaussian distribution. This process was also used for coherence spectra (Figure 2) and timing measurements (Figure 3).

To compare our MDL-based detection methods to other methods (Figure 4), we used simulated test data consisting of $N=5 \times 10^{6}$ data points generated by a two-state Markov Model with equal probability for each state. Here, the transition probability of the Markov chain was $10^{-3}$ in each time-step and the standard deviation of the Gaussian noise was equal to the step size between Markov states, giving a SNR equal to 1 . The slightly slower transitions kinetics used for test data in Figure 4 compared to the test in Figure 1 were chosen to provide test data for which the methods to be compared would all perform reasonably well. The same input time series was analyzed by the different methods. Because of the long calculation times for J-SMURF on filtered data, J_SMURF was tested on a $10^{6}$ sub segment of the full data. The $10^{6}$ segment was analyzed by Jsmurf in little more than $3 \mathrm{~h}$, while attempts to analyze the full sequence (which was five times longer) ran for more than $41 \mathrm{CPU}$ hours before the process was aborted. The histograms were rescaled for easier comparison (Figures 4B,C, cyan, and Figures 4D,E).

We then applied MDL-detection to a simulated recording of three independent ion-channels with a complex structure of subconductance states (Figure 5). Each channel had one closed state $\left(C_{0}\right)$ and 4 open states $\left(O_{1}, \ldots, O_{4}\right)$ linked according to

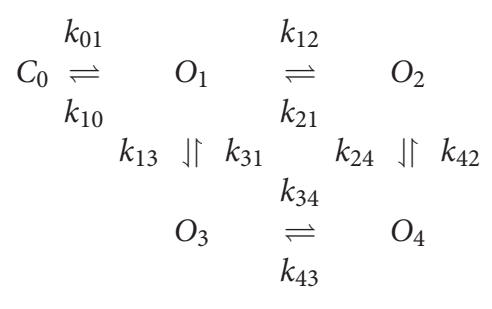

The amplitudes from the states $C_{0}, O_{1}, \ldots, O_{4}$ are $0,0.1,0.3$, 1 , and 1 . Thus, $\mathrm{O}_{3}$ and $\mathrm{O}_{4}$ are degenerate fully open states whereas $\mathrm{O}_{1}$ and $\mathrm{O}_{2}$ are nearly closed states but with some residual current. The rate constants are presented in Table 1 . The rates of transition from state $\mathrm{O}_{2}$ are relatively faster than the other rates, thus making $\mathrm{O}_{2}$ a short-lived state.

\section{Threshold-crossing, QuB, and J-SMURF Analysis of Synthetic Data}

We then tested the MDL method against a thresholdcrossing algorithm, the Viterbi algorithm as implemented in QuB (Rabiner, 1989; Nicolai and Sachs, 2013), and a jump segmentation by multiresolution filter (J-SMURF) (Hotz et al., 2013). The test was performed under optimal detection conditions for the competing algorithms: For the threshold crossing algorithm, we used prior information of the unitary steps in the process to set the detection threshold to 0.5 . We applied low-pass filtering of the time-series in order to ensure reliability of the step detection by threshold crossing. In the next step we tested the sensitivity of the threshold crossing method against low-pass filtering by applying three different filters with cutoff frequency at $1 / 20,1 / 40$, and $1 / 80$ times the Nyquist frequency (corresponding to $1,0.5$, and $0.25 \mathrm{kHz}$ if the time-series modeled a real recording sampled at $40 \mathrm{kHz}$ ). Low-pass filtering was done using a digital three pole Butterworth filter. For easier comparison with MDL, each segment between detected steps was assigned its own mean value based on the unfiltered data points in the segment. The entire step-detection algorithm was coded in MATLAB.

The same simulated data were also analyzed using the $\mathrm{QuB}$ software (Nicolai and Sachs, 2013). This approach uses the Viterbi algorithm to provide the most likely path through the state space of the HMM given the observed data. To provide the best possible conditions for event detection by this method we fitted data to the same two-state Markov model that was used to generate the test data.

In our comparison with J-SMURF we imported the Matlab test-file into R-environment using the R.matlab package. We first used "smuceR" from the R-package "stepR (Version 1.0)" to detect breaks on unfiltered data (Aspelmeier et al., 2016). In order to test jsmurf under similar conditions as in Hotz et al. (2013) we low pass filtered the data using a 4 pole Bessel filter with cut-off $=0.05$ to obtain SNR $\sim 3$. The filter characteristics were used as input parameter to the jsmurf function. We found that J-Smurf detected slightly more events than smuceR on low pass filtered data, and results from J-Smurf are reported here.

\section{QuB Analysis of hPIEZO1 Channel Unitary Current}

Data containing multiple open levels which varied in amplitude were first analyzed in QuB using the Segmental K-Means (SKM) algorithm (Qin et al., 1996b, 1997) to idealize the events. We used a model with one or two open states to find the levels. The MDL algorithm was subsequently applied to the same dataset in order to evaluate its performance against the model-based approach. In the two-state model (SKM2), the initial estimates of the current in the closed and open states were assigned manually before idealizing the time series. In the three-state model (SKM3), we included a sub-state for which we set the starting estimate to $0.4 \mathrm{pA}$. Here, the three states were connected linearly. SKM recognized all occurrences of the predefined states from the entire trace; i.e., the two-state model recognized two states (closed and open) and the three-state model recognized the presence of three states (closed, low-conductance open-state and high-conductance open-state). 


\section{A1}

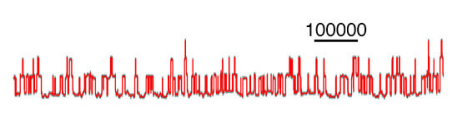

$\underline{20000}$
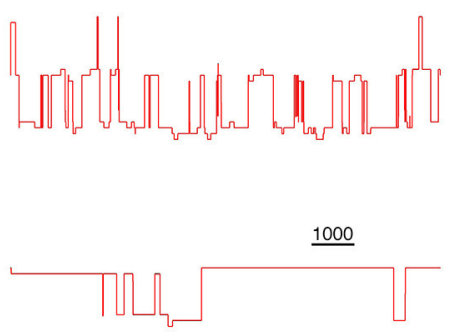

\section{B1}

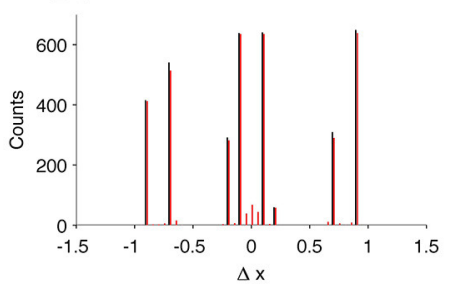

C1

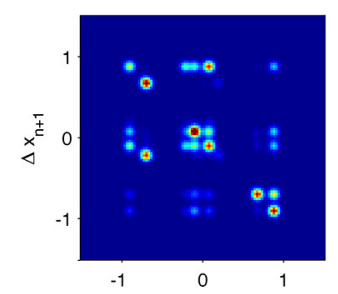

D1

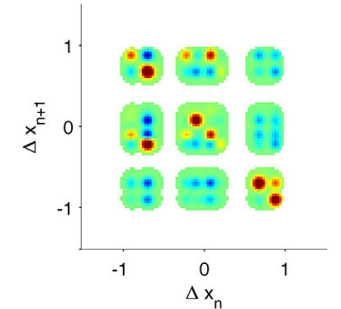

A2
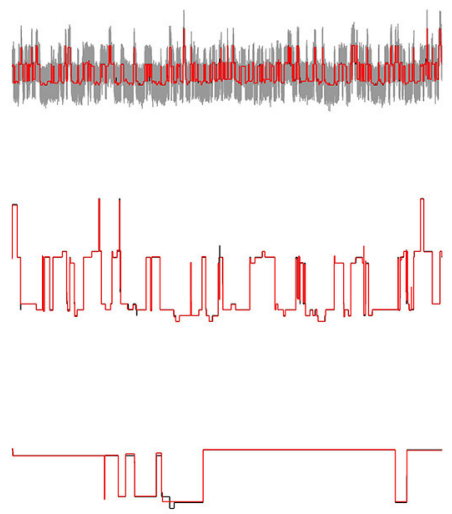

B2

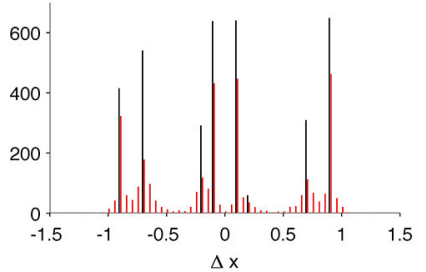

C2

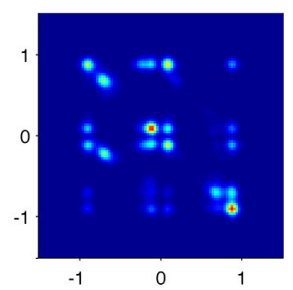

D2

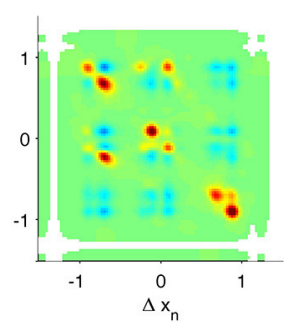

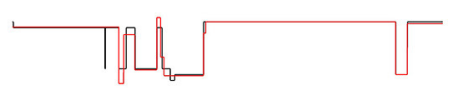
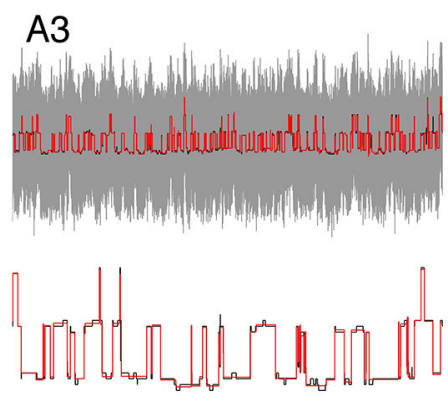

B3

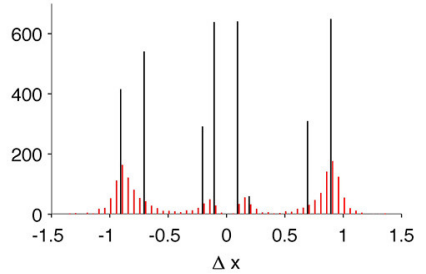

C3

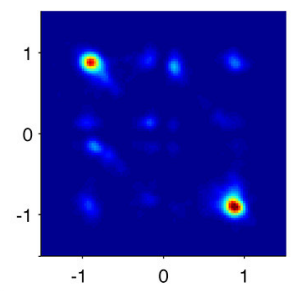

D3

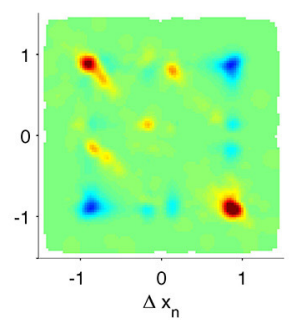

FIGURE 5 | Validation on complex simulation data with different levels of noise. Data simulates three identical, but independently operating channels. First column, (A1-D1), is the case for low noise (SNR = 33); second column, (A2-D2), is for medium noise (SNR = 3.3); and last column, (A3-D3), is for high noise $(\mathrm{SNR}=1)$. (A1-A3): Gray, segment of raw data (upper line only); black, true states; red, MDL idealization. (B1-B3): Histogram of detected steps. (C1-C3): 2D conditional histogram of neighboring steps. (D1-D3): Correlation between neighboring steps.

\section{RESULTS}

\section{Validation on Simulated Data}

We first asked how well our method resolved a simple sequence consisting of a simulated single channel embedded in Gaussian noise. The channel was modeled as a two-state Markov process of unit step-size, with equal probability of being either open or closed, and with a state transition probability of $1 / 100$, and the added Gaussian noise was set at SNR $=3.3$ or SNR $=1$ (Figure 1A top and lower panel respectively). On average, 98\% of the steps were detected at $\mathrm{SNR}=3.3$ (low noise) and only $50 \%$ at $\mathrm{SNR}=1$ (high noise). The fraction of detected steps did 
TABLE 1 | Transition kinetics of simulated complex channel used in Figure 5.

\begin{tabular}{lcc}
\hline Parameter & Value (per time-step) & Value (if sampled at $\mathbf{4 0 ~ k H z}$ ) \\
\hline$k_{01}$ & $10^{-4}$ & $4 \mathrm{~s}^{-1}$ \\
$k_{10}$ & $10^{-4}$ & $4 \mathrm{~s}^{-1}$ \\
$k_{12}$ & $10^{-5}$ & $0.4 \mathrm{~s}^{-1}$ \\
$k_{21}$ & $5 \times 10^{-3}$ & $200 \mathrm{~s}^{-1}$ \\
$k_{13}$ & $10^{-4}$ & $4 \mathrm{~s}^{-1}$ \\
$k_{31}$ & $2 \times 10^{-4}$ & $8 \mathrm{~s}^{-1}$ \\
$k_{34}$ & $2 \times 10^{-4}$ & $8 \mathrm{~s}^{-1}$ \\
$k_{43}$ & $10^{-3}$ & $40 \mathrm{~s}^{-1}$ \\
$k_{24}$ & $5 \times 10^{-3}$ & $200 \mathrm{~s}^{-1}$ \\
$k_{42}$ & $5 \times 10^{-3}$ & $200 \mathrm{~s}^{-1}$ \\
\hline
\end{tabular}

States are described by Equation (12).

not depend on record length (Figure 1B, solid green, low noise; solid blue high noise), whereas detection deteriorated in longer records when using the binary search alone, in particular at high noise levels (Figure 1B, dashed green, low noise; dashed blue high noise).

We next asked if the algorithm would generate false positive steps (Figure 1C). In general, false positives occur when a subset of consecutive data points randomly has a mean value sufficiently different from the rest of the segment to fulfill the MDL criterion. When analyzing any data set with many segments, there will be some finite probability that this occurs. To determine this probability, we constructed data series with a different total number of data points, $N$, but without deliberate introduction of any steps. For segments with $N>100$, the method detected virtually no false positive steps. The probability of erroneously dividing the segment one or more times was $6 \pm 1 \%$ for segments with $10<N<100$ (Figure 1C, black). Only in segments with $N<10$ was there any appreciable risk of detecting false positive steps. Increasing the minimum acceptable segment length, $l_{\text {min }}$, to 3 or 5 reduced the risk of false positive steps in short segments, but had negligible effect on event detection in longer segments (Figure 1C; $l_{\min }=1$, black, $l_{\min }=3$, red, $l_{\min }=5$, blue).

Thus, the risk of generating false positive steps is greater within short segments (Figure 1C). This observation has implications for application of our method for analyzing data with fast kinetics under high SNR. For example, 63\% of the segments of the input data in Figure 1A are shorter than 100 data points. Under conditions of high SNR, where the algorithm is able to detect most true transitions, some of the resulting segments can be sufficiently short to risk instances of false positive segmentation. In order to differentiate between genuine transitions and errors, we compared the expected precision (Equation 8) of the transitions (based on the length of adjacent segments) and the observed step. Under high SNR, we found that the amplitude deviated more than expected on the basis of the precision estimate in $5 \%$ of the identified transitions, and that many of these occurrences had small $|\Delta x|$ (Figure 1D, SNR = 3.3, dots indicate observed step and estimated precision. Red lines indicate 4 standard deviations from unity). In data with low SNR, the algorithm does not capture the shortest segments, and false positives will thus be rarer. Under low SNR, $4 \%$ of the transitions lay outside the expected range (Figure $1 \mathrm{E}, \mathrm{SNR}=1$ ). This was presumably because the algorithm stopped before all segments were detected. In biological data, where we do not always have prior knowledge of typical step amplitudes and kinetics, we used $l_{\min }=3$ to reduce putative false positive detections.

To verify that our method gave relevant information of the state-transitions, we calculated the joint probability histogram of neighboring steps. As the step generation mechanism was by definition a two-state Markov process (simulating a single simple channel with open and closed states), each step must necessarily be followed by an inverse step. We found that the conditional histogram showed exactly this predicted behavior: the vast majority of +1 steps (transition from closed to open state) were followed by -1 steps (transition from open to closed states) and vice versa (Figure 1F, low noise; Figure $\mathbf{1 H}$, high noise). The correlation plot revealed the Markov structure of the process: +1 steps correlated positively with “ -1 ” steps, and vice versa, whereas +1 steps correlated negatively with other +1 steps (Figure 1G, low SNR; Figure 1I, high SNR).

The above analysis showed that the algorithm resolved long segments, whereas sometimes missed short segments at low SNR. To characterize further the performance of our algorithm, we calculated the coherence spectrum between the input time series and the idealized output (Figure 2). Coherence was nearly 1 at low frequencies (indicated as inverse segment length) but decayed at high frequencies. At SNR $=1$ (Figure 2, purple), coherence was $\sim 0.5$ at frequencies corresponding to segments of length $n \sim 100$. This is consistent with our observation above that for a process with mean segment length 100 and SNR $=1, \mathrm{MDL}$ detected roughly $50 \%$ of the segments (Figure $\mathbf{1 B}$, solid blue). The observed roll-off at higher frequencies indicates that the idealized output failed to resolve shorter segments. At higher SNR the roll-off occurred at higher frequencies (higher values of $1 / n$ ), indicating that our algorithm was able to resolve increasingly finer details of the input (Figure 2; SNR 3.3, yellow; SNR 10, orange; SNR 33, blue). Thus, our algorithm resolves transient details, but in a manner limited by the SNR. It follows that our algorithm can resolve noisy data, even with SNR < 1 , if the kinetics ensure that typical segments are sufficiently long.

\section{The Combination of Binary and Tertiary Search Saves Computation Time}

We determined the computation time for MDL to resolve data as shown in Figure 1A on a typical laptop computer (Figure 3). With SNR $=3.3$ (as in Figure 1A upper), computation time scaled linearly with sequence length and was uniform across 10 trials. For example, the processing time for a sequence of 500,000 data points had a mean of 7.2 s (range 6.8-8.9 s) (Figure 3, blue circles show mean and error bars show range). With $\mathrm{SNR}=1$ (as in Figure 1A lower), the computation time was significantly higher and more variable between trials. For example, the mean processing time for 500,000 data points was $\sim 1,000 \mathrm{~s}$ (range 75-3,600 s) (Figure 3, red dots show mean and error bars show range from $n=10$ iterations). 
The computation time for uniform random data was even longer, did not vary between trials, and the processing time scaled with the square of the sequence length (Figure 3, yellow dots computation time for a single trial). To provide a comparison with HMM-based methods, we found the processing time for analyzing the sequences using our MATLAB implementation of the Viterbi algorithm to be linear and independent of SNR (Figure 3, purple asterisks show computation time for a single trial at $\mathrm{SNR}=3.3$ ).

The differences in computation time reflect which method is most involved in the search for breakpoints. While the binary search is fast, albeit apt to miss breakpoints in long segments, the tertiary search is computationally expensive, but is required to enable uniform recovery at different record lengths (Figure 1B, compare dashed and solid). However, our approach of combining binary and tertiary searches reduces computation time and enables computationally efficient searches on datasets with multiple breakpoints. However, the MDL method is much faster than J-SMURF (Hotz et al., 2013). As a comparison, JSMURF, worked for more than $3 \mathrm{~h}$ on a data sequence containing $10^{6}$ data-points. On the full $510^{6}$ data sequence J-SMURF worked for more than $41 \mathrm{CPU}$ hours before the process was aborted.

\section{Comparison of Step Detection with other Idealization Methods}

Our motivation behind developing the MDL algorithm is to enable unbiased event detection and idealization of real electrophysiological data. The algorithm is designed to idealize ion channel recordings without user-specified inputs, and with the fewest possible assumptions, thus providing a versatile and general tool. We concede that other methods of idealization might be optimal for a particular system, thus providing more information in a particular application. In order to compare the fitness of other specialized methods to MDL, we analyzed a simulation consisting of a simple two-state Markov model emitting 0 or 1 with equal probability and Gaussian noise with standard deviation 1 (Figure 4A). The transition probability between states was set to $10^{-3}$ per time step, a 10 times slower process than used above (Figures 13). The distribution of step-lengths, i.e., the dwell time in each state, followed an exponential decay function (Figures $4 \mathrm{~B}, \mathrm{C}$, black).

In this comparison of methods, we first applied thresholdbased event detection on the simulated data. The threshold was set at 0.5 , i.e., the $50 \%$ level between the two states. At the noise level used in the test, threshold-crossing required prior low-pass filtering of the data. For this, we used a 3-pole Butterworth lowpass filter, and tested the effect of three levels of low-pass filtering, i.e., at $F_{N y} / 20, F_{N y} / 40$, and $F_{N y} / 80$ (these filters correspond to 1, 0.5 , and $0.25 \mathrm{kHz}$ in relation to $40 \mathrm{kHz}$ sampling).

The algorithm's ability to detect short duration events using a threshold proved to be highly dependent on the filter width, with best sensitivity at $F_{N y} / 40$ (Figure $4 \mathrm{~A}$, dark blue, Figures $4 \mathrm{~B}, \mathrm{C}$, dark blue). With too little filtration, i.e., $F_{N y} / 20$, the threshold algorithm detected many false-positive events. On the other hand, with excessive filtration of the input data, i.e., $F_{N y} / 80$, there was a penalty in the ability of the threshold-based algorithm to detect short segments.

We next analyzed the data using QuB (Figure 4A, green). This approach uses the Viterbi algorithm, which provides the most likely sequence of states given an observed sequence of emissions, and provided that the probability of observing a particular emission from each state is known (Rabiner, 1989), without requiring prior low-pass filtering. The overall distribution of dwell times was similar to the input sequence (Figure 4B, green), although the probability of detecting transitions with a dwell time less than $\sim 30$ time-steps was lower than for the case of threshold-based detection (Figure 4C, compare green and dark blue).

Next method tested was J-SMURF (Figure 4A, cyan). This method uses a statistical multiresolution filter (Hotz et al., 2013) to provide an estimate of transitions between conductance levels with no a priori assumptions of levels and kinetics. We used the default settings of the R-implementation of J-SMURF and applied the method on data low pass filtered to obtain SNR 3, as in Hotz et al. (2013). Under these conditions, computation time for J-SMURF increased steeply with segment length and we therefore analyzed a shorter sub-segment consisting of the first $10^{6}$ datapoints. We found that J-SMURF reliably detected long segments, but missed shorter segments (Figure 4A, cyan, asterisks indicate false segmentation). In the histogram of steplengths, we found that J-SMURF did not detect segments shorter than 100 datapoints (Figures 4B,C, cyan. Note that the number of detected steps by J-SMURF is rescaled to correct for the shorter segment analyzed).

We then applied the MDL method to the data (Figure 4A, red). Overall, MDL performed well on the test data, and the distribution of dwell times matched the theoretical result (Figure 4B, red) (see also Figure 2, SNR $=1$, typical segments in the input has $n=1,000$ data points). The probability of detecting segments briefer than 30 time-steps was reduced to the same degree as seen with QuB. With MDL, there was a slight overrepresentation of segment lengths between 60 and 100, which we did not see with QuB (Figure 4C, compare red, with green and black). This is presumably due to the same effect as illustrated in Figure 1C, where short segments face a certain risk of being erroneously sub-divided. The lower detection limits observed by $\mathrm{QuB}$ and MDL are determined by the SNR of the input data, rather than being an intrinsic property of these methods.

Both MDL and J-SMURF detect steps without imposing a channel structure. The distribution of neighboring steps found using J-SMURF and MDL had a similar distribution and correlation pattern, indicating that both methods captured the main characteristic of the data. In the correlations we noticed a slightly higher incidence of off-diagonal detections by JSMURF, presumably because of missed short events (compare Figures 4D-G).

These simulation results show that it is possible to optimize detection methods by taking into account prior knowledge of the process, such as kinetics and step size. The highest temporal resolution was found with the optimally filtered threshold-based method, and the accuracy of detection of true amplitudes was 
highest with the HMM based method. JSMURF and the MDLbased detection method, on the other hand, are designed to be generally applicable. When applied to simple processes it may provide an independent confirmation of the sorts of assumptions and constraints used in more refined analyses. In these tests we found that MDL is much faster and the MDL method had superior sensitivity to detect short segments under high noise conditions as tested here.

\section{Detection of Sub-states in Multiple Channels}

Many types of data do not lend themselves to analysis by conventional methods. For example, currents arising from background channels can be present in the data recording, or individual channels can exhibit transitions between sub-states. In these cases, MDL-based detection is still reliable. As a test of our algorithm on complex data of this type, we simulated the combined output of three simultaneously active, independent, and complex ion-channels, each as given by Equation (12). The simulation generated output from states with long and short dwell times and different current amplitudes. The expected amplitude steps defined in the dataset are \pm 0.1 (from $C_{0}$ to $O_{1}$ ), \pm 0.2 (from $O_{1}$ to $O_{2}$ ), \pm 0.9 (from $O_{1}$ to $O_{3}$ ), and \pm 0.7 (from $\mathrm{O}_{2}$ to $\mathrm{O}_{4}$ ). Transitions from $\mathrm{O}_{3}$ to $\mathrm{O}_{4}$ will not give a step in current, but these two states have different transition kinetics to the other states. We analyzed the same simulated dataset with different levels of noise [Figures 5A1-A3 shows part of the test data with noise (gray), original state emissions (black), MDL idealized (red)].

The first idealization of the recordings was in a condition of low noise (Figure 5A1, black and red traces superimposed, SNR $=33$. The MDL algorithm detected multiple steps at the same amplitudes as defined for the channel $( \pm 0.9, \pm 0.7, \pm 0.2$, and \pm 0.1 ; Figure 5B1, compare black and red). There was a small fraction of false-positive detections clustered about the origin of the histogram. Based on a comparison with Figure 1C, this bias is most likely due to the favored detection of very short segments under a condition of low noise.

The complex state transitions of the simulated channels (Equation 12) were apparent in the joint probability histogram of neighboring steps. This histogram depicted numerous open events followed by closing events (Figure 5C1, along the diagonal in the lower right quadrant). and also many closing events followed by open events, Figure 5C1, along the diagonal in the upper left quadrant). In addition, numerous off-diagonal events were observed.

We see a similar pattern in the corresponding amplitude correlation map (Figure 5D1). Here, the most highly correlated transition was +0.7 , and the second most common -0.7 , and vice versa. This result is doubtless because of the rapid flickering between $\mathrm{O}_{2}$ and $\mathrm{O}_{4}$, as defined in the model. Events with a -0.1 step (transition from $O_{1}$ to $C_{0}$ ) were highly correlated with a +0.1 step (transition from $C_{0}$ to $O_{1}$ ). We also found steps of -0.7 to be highly correlated with steps of +0.7 (Figure 5D1, upper left quadrant, reflecting transitions from $\mathrm{O}_{4} \rightarrow \mathrm{O}_{2} \rightarrow \mathrm{O}_{4}$ ) and also with -0.2 transitions (reflecting $\mathrm{O}_{4} \rightarrow \mathrm{O}_{2} \rightarrow \mathrm{O}_{1}$ ), and note that the $\mathrm{O}_{4} \rightarrow \mathrm{O}_{2}$ transition seemed more strongly correlated, whereas the $\mathrm{O}_{3} \rightarrow \mathrm{O}_{1}$ transition was less correlated. These results presumably arise from the kinetics of transition away from $O_{1}$ being slower than from $\mathrm{O}_{4}$. Therefore, in the presence of other channels, slow transitions like $\mathrm{O}_{3} \rightarrow \mathrm{O}_{1} \rightarrow \mathrm{O}_{3}$ are not often observed directly in step detection, because they are likely to be interrupted by events in other channels.

We next analyzed the same simulated recording under increased noise levels (Figure 5A2, SNR = 3.3; and Figure 5A3, SNR $=1$ ). As expected, the sensitivity of detection gradually declined for small amplitude events, and the estimation of the plateau magnitudes became more influenced by the SNR (Compare red and black lines in lower panels of Figures 5A2,A3,B2,B3). The joint probability histogram and correlations between neighboring steps were remarkably robust, and most transitions observed under very low noise (Figures 5C1,D1) were also detected in recordings with moderate noise (Figures 5C2,D2). At high noise, only the most dominant amplitude events were discernable. In that condition, it was no longer possible to clearly distinguish \pm 0.7 from \pm 0.9 events. Even though the detection limit was limited by noise, it remained possible to identify $\mathrm{C}_{0} \rightarrow \mathrm{O}_{1} \rightarrow \mathrm{O}_{3}$ (Figure 5D3, small peak at $\left.\left[\Delta \mathrm{x}_{\mathrm{n}}, \Delta \mathrm{x}_{\mathrm{n}+1}\right]=[0.1,0.9]\right), O_{1} \rightarrow C_{0} \rightarrow O_{1}$ (Figure 5D3, small peak at $\left.\left[\Delta \mathrm{x}_{\mathrm{n}}, \Delta \mathrm{x}_{\mathrm{n}+1}\right]=[-0.1,0.1]\right)$, and $\mathrm{O}_{3} \rightarrow \mathrm{O}_{1} \rightarrow C_{0}$ (Figure 5D3, small peak at $\left[\Delta \mathrm{x}_{\mathrm{n}}, \Delta \mathrm{x}_{n+1}\right]=$ $[-0.9,-0.1])$.

Thus, our analysis of simulated recordings predicts that the algorithm should successfully resolve subconductance structures even under high noise conditions and in the presence of potentially interfering signals arising from multiple channels.

\section{Analysis of Human PIEZO Channels}

We tested the MDL method for analysis of experimental data from human PIEZO1 ion channels. The PIEZO1 channel is a mechanosensitive receptor that is activated by membrane tension (Cox et al., 2016), which can be applied experimentally by stretching patched membranes with either positive or negative pressure (Besch et al., 2002). This channel activates and deactivates rapidly, tracking closely the onset and termination of the pressure stimulus. It also shows voltage-dependent inactivation, which is faster at hyperpolarized membrane potentials. In our preparation we observed openings to multiple levels during application of steady pressure; some of these open levels may in fact represent sub-conductance states of the channel, affording a useful test of the performance of our MDL algorithm.

The analyzed segment lasted $140 \mathrm{~s}$ and contained 14 episodes of applied stimuli, each of $5 \mathrm{~s}$ duration, with a $5 \mathrm{~s}$ relaxation interval between the stimuli. We held the patch at five different potentials during the recording (Figure 6A shows the voltages and Figure 6 $\mathbf{B}$ the suction pulse train that served as the mechanical stimulus; Figure 6C shows the recorded current). The drift in the baseline leakage current resulting from changes in voltage was canceled using the baseline algorithms in $\mathrm{QuB}$ (http://www.qub.buffalo.edu), whereas the MDL method was applied to the unfiltered current trace. MDL detected the channel opening events even though the unitary current amplitude changed as a function of the potential applied across the patch 


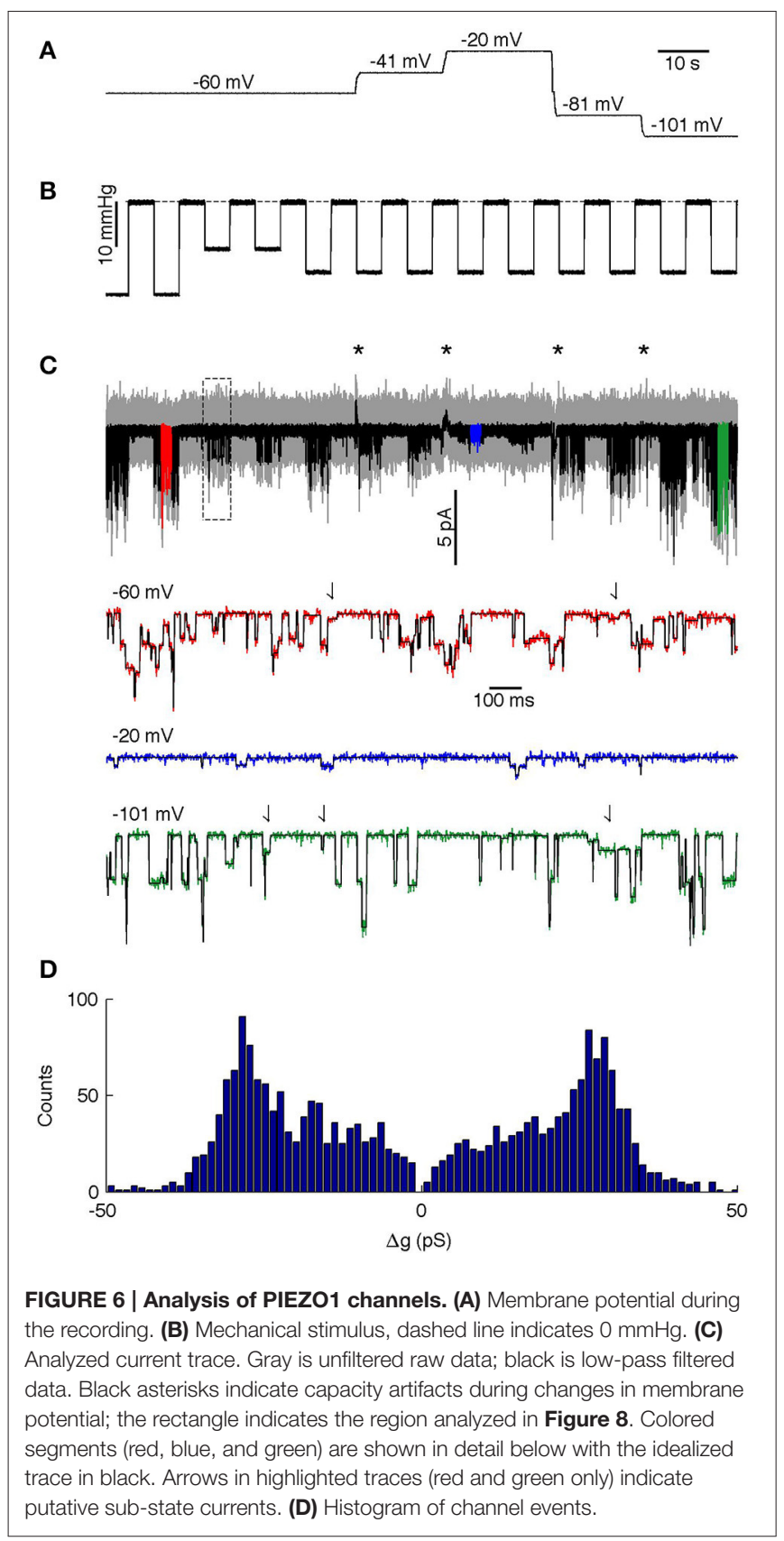

(Figure 6C, compare amplitude of steps in red, blue, and green highlights). The MDL algorithm also detected current steps of multiple sizes (Figure 6C, putative sub-state currents indicated by arrows in red and green highlights). If the membrane potential was $-60 \mathrm{mV}$ or more negative, the current steps appeared to reflect openings of different duration and current (Figure 6C, arrows in red and green highlight indicate small current events). At low polarization $(-20 \mathrm{mV})$, these events were detected only rarely, presumably because their amplitude fell below the noise detection limit. The algorithm detected 125, 191, 985, 230, and 632 events at $-20,-40,-60,-80$, and $-100 \mathrm{mV}$ respectively. A total of 78 events that were detected in proximity to recording artifacts occurring at changes in holding potential were ignored in the analysis (see asterisks in Figure 6C).

To facilitate analysis across the different voltages of the time series, we divided the MDL-detected current steps by the concurrent holding potential to calculate changes in conductance, $\Delta g$. assuming channel conductance is linear. The event amplitude histogram presented a wide range of conductance steps with peaks at $\Delta g= \pm 28 \mathrm{pS}$, but we also observed smaller transitions down to $\Delta g= \pm 6 \mathrm{pS}$ (Figure 6D). The relative fraction of smaller conductance steps varied with the holding potential. Thus, at -20 and $-40 \mathrm{mV}$ the algorithm detected relatively few steps with amplitude lower than $28 \mathrm{pS}$. However, higher holding potentials revealed a wide distribution of different amplitudes, although steps at $\pm 28 \mathrm{pS}$ amplitude were always present and appeared to be the dominant amplitude. This is similar to previous studies showing that the chord conductance (at $-80 \mathrm{mV}$ ) of hPIEZO1 channels in the presence of $80 \mathrm{mM}$ TEA is $31 \mathrm{pS}$ (Gnanasambandam et al., 2015).

We then analyzed the joint probability distribution and correlation of neighboring steps at different holding potentials (Figures 7A,B shows $-60 \mathrm{mV}$, Figures 7C,D shows $-100 \mathrm{mV}$ ). The main transitions at $\pm 28 \mathrm{pS}$ were clearly identified at all holding potentials (Figures 7A,C shows -60 and $-100 \mathrm{mV}$, other holding potentials not shown). However, there were some notable differences as a function of the voltage. For example, at $-60 \mathrm{mV}$ the $+6 \mathrm{pS}$ open steps were frequently followed by a $-6 \mathrm{pS}$ closing step, whereas other sub-states were infrequent. However, at $-100 \mathrm{mV}$ holding potential there was a wider range of sub-amplitude openings, including \pm 10 and $\pm 16 \mathrm{pS}$.

Multiple factors must account for the details of the observed channel kinetics, and a full characterization of hPIEZO1 channel properties is beyond the scope of this report. For the present, we emphasize that the unsupervised MDL idealization and our joint probability and amplitude correlation method proved to be sensitive to the changes in the full open amplitudes and substates. Thus, our method may provide a firm foundation for subsequent analysis using this model approach.

Several methods are already available for the analysis of single-channel data. To get an understanding of performance of the MDL method in comparison to the established techniques, we analyzed a segment of the hPIEZO1 data with QuB using the Segmental K-means (SKM) method to estimate the unitary channel amplitudes. Unlike MDL, this method explicitly employs a defined 2- or 3-state model, with user-defined rates emulating the inverses of the observed closed and open state lifetimes. We chose an arbitrary part of the recorded segment for the MDL analysis (Figure 8A, indicated by the rectangular inset in Figure 6C). The segment was recorded at $-60 \mathrm{mV}$ holding potential, where \pm 6 and $\pm 28 \mathrm{pS}$ were the dominant transitions according to the analysis above (Figures 7A,B).

We first idealized the segment using SKM for a 2-state ion channel. The idealized current trace contained 40 open or closed events. The analysis yielded a transition between the closed state and a single open state of $29.8 \mathrm{pS}$ conductance. Visual inspection confirmed that the idealization detected the high amplitude openings but missed a number of smaller amplitude openings (Figure 8B, compare SKM 2 and SKM 3 or MDL). 

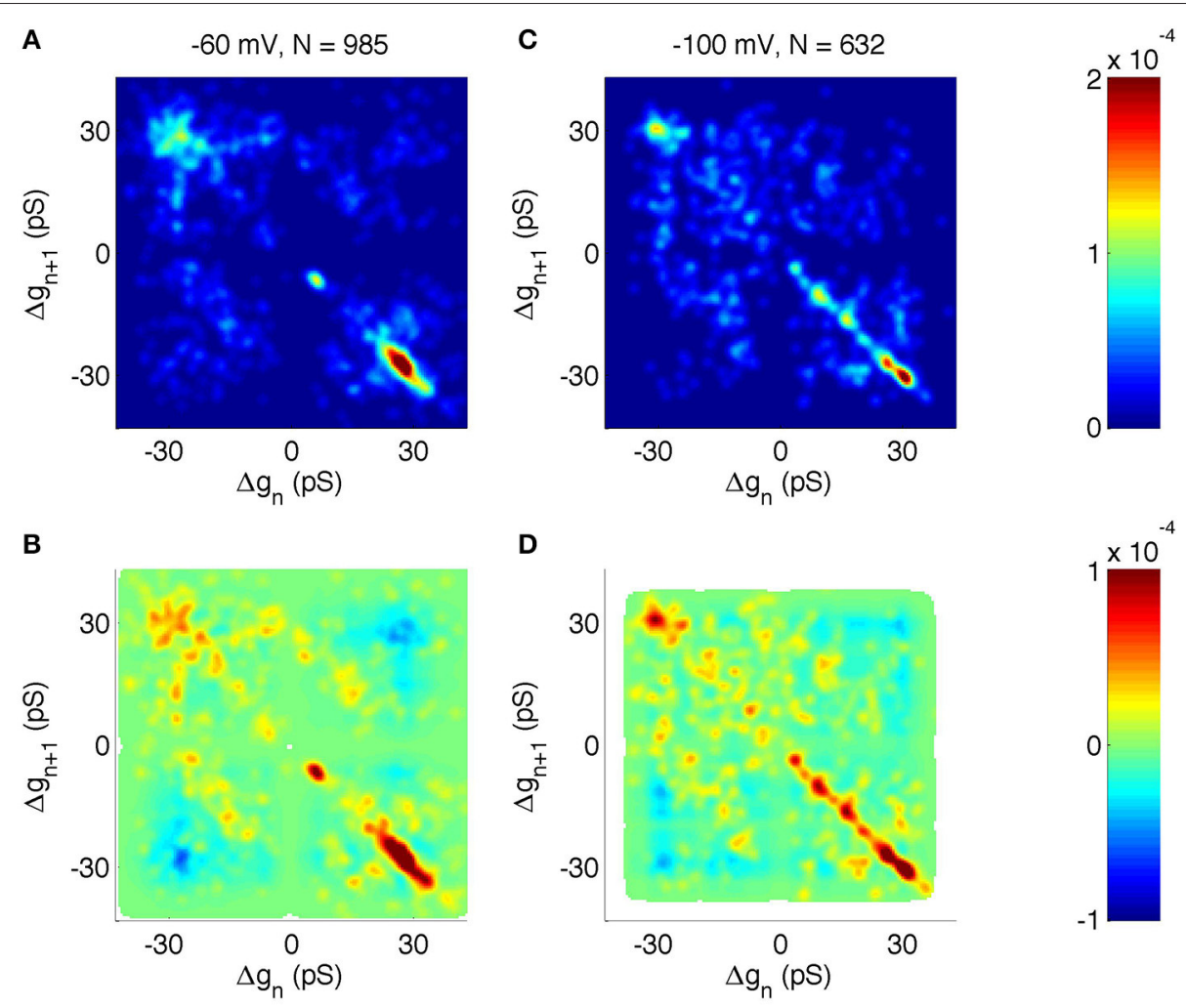

FIGURE 7 | Correlation analysis of neighboring events in PIEZO1 channels recorded at -60 and $-100 \mathbf{m V}$ holding potentials. As in other figures, $x$ and $y$ scales are arranged so that open events followed by closed events populate the lower right quadrant. (A) Distribution of neighboring events at $-60 \mathrm{mV}$. The $x$-axis shows $\Delta g$ of event $n$ and the $y$-axis shows $\Delta g$ of the following event, $n+1$. (B) Correlation of neighboring events at $-60 \mathrm{mV}$. Red indicates neighboring events occurring more frequently than random. Blue indicates neighboring events occurring less frequently than random. White areas indicate undefined correlations. (C, D) same as (A,B) but for $-100 \mathrm{mV}$ holding potential.

When the segment was reanalyzed with SKM using a 3-state linearly-connected model, the idealized data contained 86 events. Now the event sizes were either $\Delta g= \pm 6.7 \mathrm{pS}$ or \pm 23.2 pS. The current at the fully opened state was $29.8 \mathrm{pS}$, equal to the sum of the two main event sizes. By visual inspection, the smaller event size appeared to reflect opening to the same subconductance state discussed above (see arrows in highlighted parts of Figure 6C).

We then idealized the data segment using the MDL method, which detected 61 events and proved to be sensitive to most of the visually-observed openings to both the sub-state and the fullyopen state. The apparent amplitude of the events was roughly the same as for the 3-state SKM approach, although MDL appeared slightly conservative, in failing to capture some possible small amplitude events detected by the 3-state model (Figure 8B).

\section{DISCUSSION}

Analysis of currents from a membrane patch containing an unknown number of channels in the presence of a noisy background or leakage current is challenging, regardless of the applied algorithm. The various available methods all have different strengths and weaknesses. Most of the currently used methods require some degree of user-dependent input and supervision, through selection of a low-pass filter followed by application of an event-detection threshold, or a priori Markov models. While such methods can have good performance in analyzing records with a few simple channels, there is currently no way of accommodating complicated records, which may contain events of differing amplitudes and kinetics. While the HMM based methods use a kinetic model to estimate the individual channel current amplitudes, the observed amplitudes are relatively insensitive to the magnitude of the transition rate constants (Qin et al., 1996a). For dealing better with multichannel currents from identical channels, the MAC (macroscopic) algorithm in QuB (Milescu et al., 2005) is robust for estimating transition kinetics, the number of active channels in the pool, and the mean jump size arising from an arbitrary stimulus (Bae et al., 2013).

Here we show that the Minimum Description Length principle $(\mathrm{MDL})$ enables idealization of patch recordings in a user-independent manner. We find that this method can be applied to raw data without the need to correct baselines, and has good performance even in cases where event amplitude changes during the recording (Figures 6, 7). We provide proof of this principle based on three main findings. First, we applied 


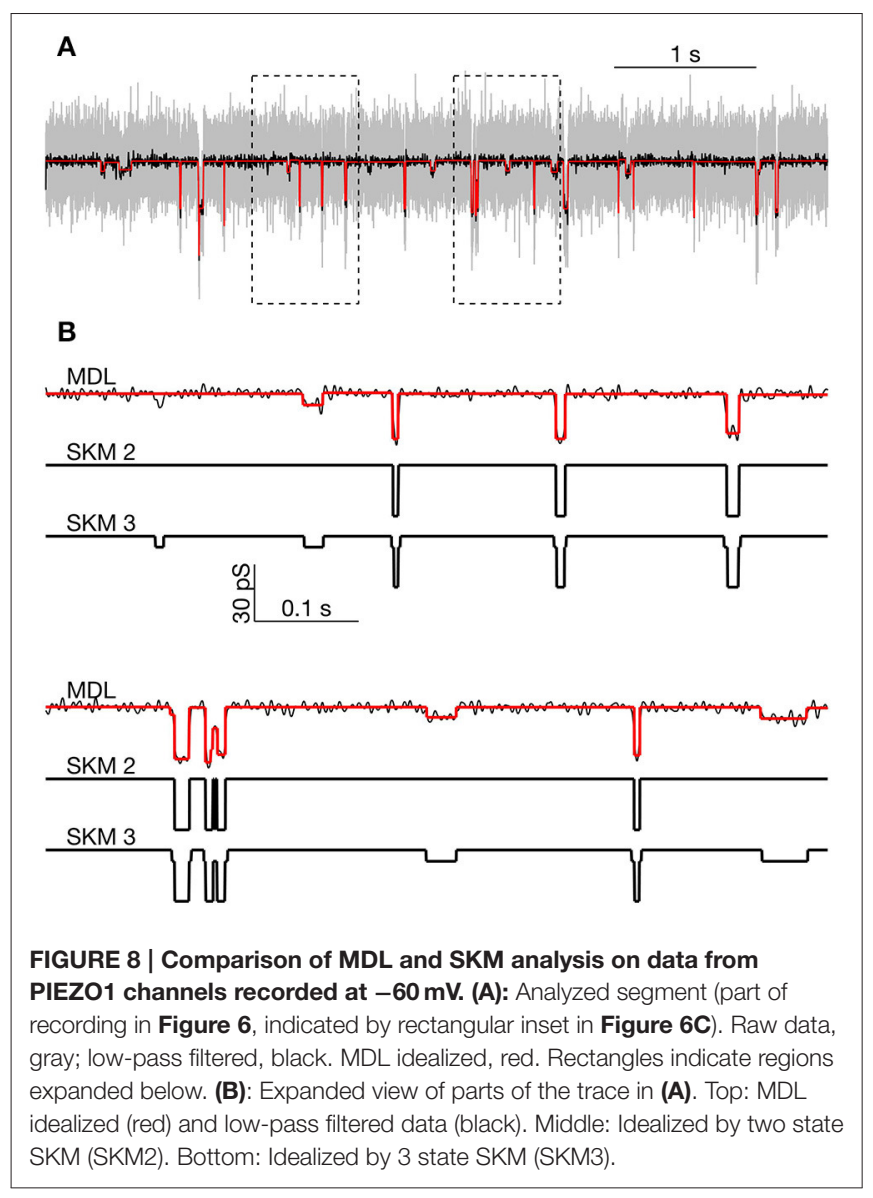

our method to simulated data and investigated the limits for detection. These simulations showed our method to be efficient for detecting events with multiple independent Markov processes under various noise levels. Finally, we used a novel correlation analysis to relate the results to the transitions of single channels.

Our approach required the solution of two sub-problems: First, we needed an objective criterion for model selection. More precisely, it was necessary to test whether a complex model of the data using many discrete segments is superior to a simple model with fewer segments. For this type of assessment, several measures of quality have been proposed, among which the Akaike Information Criterion (Akaike, 1973), Bayes Inference Criterion (Schwarz, 1978), and the Minimum Description Length Principle (Rissanen, 1978; Lee, 2001). In our hands, MDL proved to work best, and we derived a cost function that described the balance between model complexity and fitness. Optimizing the cost function posed a second challenge: Calculation of the description length depends on multiple interdependent variables, all of which contribute to the cost function. A full search of all possible combinations in solution-space is not universally applicable, since the expected number of events in real data is often in the thousands, which would be computationally impossible. Instead, we resorted to an iterative procedure in which breakpoints were inserted sequentially. One method for this is presented by the binary segmentation process (Scott and
Knott, 1974; Kalafut and Visscher, 2008). However, when a binary search was applied to simulated data, the fraction of detected steps declined as a function of record length. In contrast, no such dependence was observed when implementing a tertiary search, where the algorithm searches for possible combinations of two break points before terminating (compare solid to dashed lines in Figure 1B). A binary search was used in the method presented earlier by Kalafut and Visscher (2008) for analyzing movement of the molecular motor kinesin. Presumably, loss of detection in long time series is less of a concern in their application because the motion of kinesin has a preferred direction, which makes it simpler to decompose the time series into segments. For ion channel applications, time series are often generated by a stationary random process, which makes it more unlikely that breakpoints inserted one by one fulfills the criterion given by Equation (4). Thus, resolution of typical data for our application also requires the more elaborate tertiary search method where breakpoints are inserted two by two. However, to optimize the computing time and reliability, our algorithm combines binary and tertiary searches (Figure 3).

We anticipate that there could be cases for which the tertiary search method will also be sub-optimal. For example, a long time-series constructed of alternating segments of equal length would require a higher SNR in order to be resolved than would be necessary if the segments were of random length, as in our tests. However, this situation is unlikely to occur in real data from ion channels for which stochastic processes drive the changes in conductance states. Furthermore, were such a circumstance actually encountered, there are convenient analysis methods for accommodating periodicity (Little et al., 2011).

Proper use of prior knowledge of the underlying biophysical processes generally enhances data analysis. For example, supervised methods such as SCAN take into account the presence of low-pass filters, and are thus able to determine transition points with accuracy exceeding the sampling rate of the data (Colquhoun and Sakmann, 1985). We found that the MDL method approached (but did not exceed) the performance of methods that explicitly use prior information (Figure 4). In situations where analysis can be performed by more specialized methods, the MDL-based method may nevertheless serve as an independent test of the model assumptions, and guide development of more refined analysis. Due to the minimal number of assumptions, our unsupervised MDL method may prove particularly useful for complex data from recordings of channels with unknown sub-state structure, and in cases where multiple independent channels are active (Figures 5, 8). We find that the J-SMURF method resembles our method the most, because it aims to idealize ion-channel data without imposing a particular channel structure (Hotz et al., 2013). When running with default settings, J-SMURF does not require user inputs. However we found that low-pass filtering the test sequence improved event detection, although at the expense of computation time. In our comparison we found that MDL was much faster in analyzing long records and was more sensitive to short segments (Figure 4).

However it is worth mentioning that the MDL method performs best with white noise. In the current version of the 
MDL algorithm, colored noise could lead to false segmentation and one may have to decimate the data in order to decrease correlation between adjacent data points. The capability of JSMURF to handle colored noise is an obvious advantage, in particular if the filter characteristics are known. Hidden markov models have also been applied to low-pass filtered data and may also provide an alternative if the state structure of the channel is known (Venkataramanan and Sigworth, 2002).

We applied the MDL method to experimental recordings of human PIEZO1 (hPIEZO1) channels, in which we encountered currents with multiple amplitudes (Figures 6, 7). Our analysis showed that the algorithm has particular advantage in situations where prior knowledge of the sub-conductance states of a channel is lacking. The flexibility of the MDL algorithm is a desirable property when performing analyses on large datasets. The MDL algorithm is generalized and can be used with timeseries datasets acquired from other disparate sub-fields of biology characterized by state models (Nicolai and Sachs, 2014).

\section{CONCLUSION}

We developed an idealization method based on the minimal description length principle for the use of analyzing ion channel recordings. The method was validated on simulated data, with

\section{REFERENCES}

Akaike, H. (1973). "Information theory and an extension of the maximum likelihood principle," in Proceedings of the 2nd International Symposium on Information Theory.

Aspelmeier, T., Hotz, T., Sieling, H., and Florian, P. (2016). stepR: Fitting StepFunctions. R package version 1.0-6. Available online at: https://CRAN.R-project. org/package $=$ stepR

Bae, C., Gnanasambandam, R., Nicolai, C., Sachs, F., and Gottlieb, P. A. (2013). Xerocytosis is caused by mutations that alter the kinetics of the mechanosensitive channel PIEZO1. Proc Natl. Acad. Sci. U.S.A. 110, E1162E1168. doi: 10.1073/pnas.1219777110

Besch, S. R., Suchyna, T., and Sachs, F. (2002). High-speed pressure clamp. Pflugers Arch. 445, 161-166. doi: 10.1007/s00424-002-0903-0

Carter, B. C., Vershinin, M., and Gross, S. P. (2008). A comparison of step-detection methods: how well can you do? Biophys. J. 94, 306-319. doi: 10.1529/biophysj.107.110601

Chan, T. F., Golub, G. H., and Leveque, R. J. (1983). Algorithms for computing the sample variance: analysis and recommendations. Am. Stat. 37, 242-247. doi: 10.1080/00031305.1983.10483115

Colquhoun, D., and Sakmann, B. (1985). Fast events in single-channel currents activated by acetylcholine and its analogues at the frog muscle end-plate. J. Physiol. 369, 501-557. doi: 10.1113/jphysiol.1985.sp015912

Colquhoun, D., and Sigworth, F. J. (1995). "Fitting and statistical analysis of singlechannel records," in Single-Channel Recording, 2nd Edn., eds B. Sakmann and E. Neher (New York, NY: Springer), 483-587.

Cox, C. D., Bae, C., Ziegler, L., Hartley, S., Nikolova-Krstevski, V., Rohde, P. R., et al. (2016). Removal of the mechanoprotective influence of the cytoskeleton reveals PIEZO1 is gated by bilayer tension. Nat. Commun. 7:10366. doi: $10.1038 /$ ncomms 10366

Davis, R. A., Lee, T. C. M., and Rodriguez-Yam, G. A. (2006). Structural break estimation for nonstationary time series models. J. Am. Stat. Assoc. 101, 223-239. doi: 10.1198/016214505000000745

Dreyer, J. K. (2016). Scan for Breaks [Online]. Matlab Central, File ID: \#60224. Available online at: http://www.mathworks.com/matlabcentral/fileexchange/ 60224-scan-for-breaks characterization of event detection reliability under different noise and recording conditions. We the applied the algorithm to the analysis of patch clamp recordings of currents from the human PIEZO1 channel. Results of this test confirmed the algorithm's fitness to detect sub-conductance states.

\section{AUTHOR CONTRIBUTIONS}

RG, MN, JH, FS, and JD analyzed data. RG, JH, and MN provided experimental data. JD designed algorithm and wrote matlab code. $\mathrm{RG}, \mathrm{MN}, \mathrm{CN}, \mathrm{FS}$, and JD wrote the paper.

\section{ACKNOWLEDGMENTS}

This study was supported by the Lundbeck Foundation and the Dynamical Systems Interdisciplinary Network, University of Copenhagen (JD), the Novo Nordisk Foundation, and the Danish National Research Foundation (JH, MN) and the NIH (FS, RS) R01HL054887. We note professional editing of the manuscript by Inglewood Biomedical Editing. MATLAB version of the MDLalgorithm is available at the File Exchange at MATLAB Central (Dreyer, 2016), and the algorithm is also available as a plug-in for the ion-channel analysis program QuB (Nicolai and Sachs, 2013).

Gnanasambandam, R., Bae, C., Gottlieb, P. A., and Sachs, F. (2015). Ionic selectivity and permeation properties of human PIEZO1 channels. PLoS ONE 10:e0125503. doi: 10.1371/journal.pone.0125503

Gottlieb, P. A., Bae, C., and Sachs, F. (2012). Gating the mechanical channel Piezo1: a comparison between whole-cell and patch recording. Channels (Austin) 6, 282-289. doi: 10.4161/chan.21064

Hotz, T., Schutte, O. M., Sieling, H., Polupanow, T., Diederichsen, U., Steinem, C., et al. (2013). Idealizing ion channel recordings by a jump segmentation multiresolution filter. IEEE Trans. Nanobiosci. 12, 376-386. doi: 10.1109/TNB.2013.2284063

Kalafut, B. S., and Visscher, K. (2008). An objective, model-independent method for detection of non-uniform steps in noisy signals. Comput. Phys. Commun. 179, 716-723. doi: 10.1016/j.cpc.2008.06.008

Killick, R., Fearnhead, P., and Eckley, I. A. (2012). Optimal detection of changepoints with a linear computational cost. J. Am. Stat. Assoc. 107, 1590-1598. doi: 10.1080/01621459.2012.737745

Lee, T. C. M. (2001). An introduction to coding theory and the twopart minimum description length principle. Int. Stat. Rev. 69, 169-183. doi: 10.1111/j.1751-5823.2001.tb00455.x

Little, M. A., Steel, B. C., Bai, F., Sowa, Y., Bilyard, T., Mueller, D. M., et al. (2011). Steps and bumps: precision extraction of discrete states of molecular machines. Biophys. J. 101, 477-485. doi: 10.1016/j.bpj.2011.05.070

Milescu, L. S., Akk, G., and Sachs, F. (2005). Maximum likelihood estimation of ion channel kinetics from macroscopic currents. Biophys. J. 88, 2494-2515. doi: 10.1529/biophysj.104.053256

Murphy, K. (1998). Hidden Markov Model (HMM) Toolbox for Matlab [Online]. Available online at: http://www.cs.ubc.ca/ murphyk/Software/HMM/hmm. html (Accessed February 26, 2016).

Nicolai, C., and Sachs, F. (2013). SOLVING ION CHANNEL KINETICS WITH THE QuB SOFTWARE. Biophys. Rev. Lett. 8, 191-211. doi: 10.1142/S1793048013300053

Nicolai, C., and Sachs, F. (2014). Fitting random data to state models with QuB software. Biophys. Rev. 8, 191-211. doi: 10.1142/S1793048013300053

Parsons, S. P., and Huizinga, J. D. (2013). Statistical assessment of change point detectors for single molecule kinetic analysis. J. Membr. Biol. 246, 407-420. doi: 10.1007/s00232-013-9553-8 
Qin, F. (2007). Principles of single-channel kinetic analysis. Methods Mol. Biol. 403, 253-286. doi: 10.1007/978-1-59745-529-9_17

Qin, F., Auerbach, A., and Sachs, F. (1996a). Estimating single-channel kinetic parameters from idealized patch-clamp data containing missed events. Biophys. J. 70, 264-280. doi: 10.1016/S0006-3495(96)79568-1

Qin, F., Auerbach, A., and Sachs, F. (1996b). Idealization of single-channel currents using the segmental K-means method. Biophys. J. 70, Mp432.

Qin, F., Auerbach, A., and Sachs, F. (1997). Maximum likelihood estimation of aggregated Markov processes. Proc. Biol. Sci. 264, 375-383. doi: 10.1098/rspb.1997.0054

Rabiner, L. R. (1989). A tutorial on hidden Markov-models and selected applications in speech recognition. Proc. IEEE 77, 257-286. doi: 10.1109/5. 18626

Rissanen, J. (1978). Modeling by shortest data description. Automatica 14, 465-471. doi: 10.1016/0005-1098(78)90005-5

Schwarz, G. (1978). Estimating dimension of a model. Ann. Stat. 6, 461-464. doi: $10.1214 /$ aos/1176344136

Scott, A., and Knott, M. (1974). A cluster analysis method for grouping means in the analysis of variance. Biometrics 30, 507-512. doi: 10.2307/ 2529204

Suchyna, T. M., Markin, V. S., and Sachs, F. (2009). Biophysics and structure of the patch and the gigaseal. Biophys. J. 97, 738-747. doi: 10.1016/j.bpj.2009.05.018
Vandongen, A. M. (1996). A new algorithm for idealizing single ion channel data containing multiple unknown conductance levels. Biophys. J. 70, 1303-1315. doi: 10.1016/S0006-3495(96)79687-X

Venkataramanan, L., and Sigworth, F. J. (2002). Applying hidden Markov models to the analysis of single ion channel activity. Biophys. J. 82, 1930-1942. doi: 10.1016/S0006-3495(02)75542-2

Conflict of Interest Statement: The authors declare that the research was conducted in the absence of any commercial or financial relationships that could be construed as a potential conflict of interest.

The reviewer RR and handling Editor declared their shared affiliation, and the handling Editor states that the process nevertheless met the standards of a fair and objective review.

Copyright ( 2017 Gnanasambandam, Nielsen, Nicolai, Sachs, Hofgaard and Dreyer. This is an open-access article distributed under the terms of the Creative Commons Attribution License (CC BY). The use, distribution or reproduction in other forums is permitted, provided the original author(s) or licensor are credited and that the original publication in this journal is cited, in accordance with accepted academic practice. No use, distribution or reproduction is permitted which does not comply with these terms. 